\title{
The effect of overtraining on reversal shift behavior in rats reinforced with electrical brain stimulation
}

\author{
PHILIP G. MEAD \\ Keuka College, Keuka Park. New York 14478
}

\begin{abstract}
Ten naive male Long-Evans rats were reinforced with intracranial stimulation (ICS) during a brightness discrimination reversal problem. Half of these animals were trained to criterion prior to receiving a reversal shift, while the other half were given an additional 100 postcriterion (overtraining) trials before being presented with the reversal shift. Rats in the overtraining group required significantly more trials to master the reversal shift than those in the nonovertraining group. These findings are discussed within the context of the selective attention model of discrimination learning. Related ICS studies are also considered.
\end{abstract}

Over the past 20 years, numerous experiments have dealt with the examination of parameters influencing reversal shift behavior. Reid (1953) reported that rats given 150 overtraining trials beyond criterion learned a reversal shift faster than those not receiving the extra amount. Since then, many investigators have attempted to examine the various factors associated with what is now called the "overtraining reversal effect" (ORE). Perhaps the most extensive series of studies has been performed by Mackintosh (1965), who recently reviewed many of the factors considered influential in the ORE phenomenon (Mackintosh, 1969). One proposition receiving substantial support was that obtainment of an ORE was maximized in situations involving both a difficult task and a large (conventional) reward. Since several investigators (Johnson, 1968; Keesey, 1966; Lindholm \& Keesey, 1970) have reported that electrical brain stimulation can serve as a strong reinforcer in the learning of discrimination tasks, it was hypothesized that rats might demonstrate a substantial ORE if reinforced by such conditions while performing a brightness discrimination similar to that employed by Mackintosh (1969, Experiment 1). Such a finding would thereby provide more generality to the ORE which, to date, has been a rather elusive form of behavior.

\section{METHOD}

\section{Subjects}

Bipolar stainless steel electrodes were stereotaxically implanted in 27 adult male Long-Evans rats. The electrodes were aimed at the posterior hypothalamus and secured to the skull by stainless steel screws and dental cement. Following a 1-week recovery period, self-stimulation rates across a range of current intensities (0-500 microamps) were recorded from each animal. Ten rats were observed to maintain a barpress rate of at least 60 presses $/ \mathrm{min}$ for $5 \mathrm{~min}$, and these animals were selected to participate in the study. Five were randomly placed in each of two treatment conditions, an overtrained (OT) group and a nonovertrained (NOT) group.

\section{Apparatus}

A Grice-type discrimination box, similar to that employed by Mackintosh (1969. Experiment 1), was used. The $12 \times 6$ in. goalboxes were painted either light or dark gray, with either vertical or horizontal black stripes.

Electrical brain stimulation was delivered by a stimulator which produced 60-cycle sine wave current of $.5 \mathrm{sec}$ train duration, contingent upon pressing the bar in the correct goalbox. Current level was monitored with a Tectronix oscilloscope, while stimulus parameters and response patterns were programmed using standard relay equipment. During training and testing, the room was completely dark except for a 75-W lamp placed directly above the discrimination box. Outside noises were masked by a white noise generator.

Procedure

On Day 1, all rats were tested in a separate box to determine the current level necessary to maintain a barpress rate of at least $60 \mathrm{responses} / \mathrm{min}$ for a 5 -min period. This current level was later employed as the rewarding ICS during the discrimination task. During Day 2, each animal was allowed 100 reinforced barpresses in each of the goalboxes. the order of presentation varied to overcome the development of a preference for any box. The same procedure was followed on Day 3. except that after every 20 th response, the animal was lifted backwards toward the startbox until each rat was finally observed to run the length of the apparatus to obtain brain stimulation. All testing occurred on Day 4. For the initial discrimination problem. the light gray box was made the correct choice for all rats, regardless of stripe orientation, and reinforcement consisted of the opportunity for 15 barpresses, each delivering a .5-sec train of ICS. On any single trial, both attributes of each dimension were present. Goalbox locations were determined by a Gellermann series, a 5-sec intertrial interval was employed, and the learning criterion was set at 9 out of 10 correct trials. using a correction procedure. Upon reaching criterion. those animals in the OT group were given an additional 100 trials prior to reversal. while the NOT group received an immediate reversal shift.

\section{RESULTS}

The trials to criterion scores for each group on both the original and reversal tasks are shown in Table 1. These data clearly reveal that both groups performed similarly on their original learning task, but differed markedly on the reversal. In order to evaluate the reliability of these findings, a two-way analysis of variance was performed. Here, it was found that the OT group had significantly slower reversal acquisition scores than the NOT group $(F=18.65$, df $=1, p<.01)$. No significant differences were obtained across groups 
Table 1

Trials to Criterion Scores for the OT and NOT Groups on Original and Reversal Tasks

\begin{tabular}{clcc}
\hline & & \multicolumn{2}{c}{ Discrimination } \\
\cline { 3 - 4 } Group & & Original & Reversal \\
\hline \multirow{4}{*}{ Overtrained } & T & 246.0 & 447.0 \\
& Mean & 49.2 & 89.4 \\
& SD & 19.7 & 25.1 \\
& T & 210.0 & 285.0 \\
& Nonovertrained & 42.0 & 57.0 \\
& SD & 26.7 & 27.8 \\
\hline
\end{tabular}

between scores on the original task vs those on the reversal task, nor was a significant interaction between groups and tasks obtained.

\section{DISCUSSION}

The present experiment demonstrates that overtraining serves to retard acquisition of a brightness reversal problem in rats rewarded with ICS. Animals receiving only criterion training learned a brightness reversal shift significantly faster than those given 100 postcriterion trials. These findings are exactly opposite what was originally predicted according to the previously discussed ORE data, and suggest that an interaction may exist between the type of reward and training conditions employed. It is possible that behaviors rewarded with ICS are more resistant to change than those rewarded with food. Kling and Matsumiya (1962), for example, demonstrated that animals receiving ICS reinforcement on the original problem reversed more slowly than those trained with a food reward. Within the present experiment, however, such comparisons are impossible, since no food reward group was employed. It can only be surmised that those animals in the OT group were at a disadvantage when presented with the reversal of the problem on which they had received the additional training, and that this disadvantage may be due to certain aspects inherent in the electrical brain stimulation employed. It should be indicated, however, that similar studies employing conventional rewards (Mackintosh, 1969, Experiments 2 and 3) differ not only in the type of reinforcement used, but also in various procedural aspects, such as training conditions and the manner in which reinforcement is delivered. As such, a precise statement on the source of impaired reversal scores under ICS conditions compared to those under food conditions remains open to speculation. On the one hand, the high incentive properties known to accompany ICS reinforcement (Gallistel, 1966) may have interfered somehow with the animals' ability to learn the brightness discrimination to any great degree. Within the framework of the selective attention model, overtrained Ss in the study may not have learned to attend to the relevant stimulus dimension at the time of reversal. at least to the extent exhibited by the NOT group. Perhaps the intensity of reinforcement of the ICS prevented the OT Ss from learning about the appropriate stimulus dimension (brightness) used. It would appear difficult, however, for such an explanation to predict the significant negative effect actually found in this group. An alternative approach might be to propose that overtraining provided much greater strength to the response attachments of such Ss, in contrast to those not receiving the additional trials, and thereby made extinction of such response attachments and attention to the appropriate stimulus dimension more difficult at the time of reversal. If the formation of response attachments (Stage 1 in the selective attention model) could be considered a less difficult task than the "switching in" of the relevant analyzer (Stage 2), then the present findings appear to fit quite well with Denenberg's (1964) hypothesis on the theoretical relationships between emotional reactivity and adult performance across tasks of varying difficulty. According to Denenberg (1964), highly aroused organisms should perform poorly when attempting difficult tasks, but should do well when given easy tasks; thus, the energizing consequences of ICS in the immediate investigation may have facilitated acquisition of the initial response attachments, but proved detrimental ultimately in learning about the appropriate dimension analyzer. Some additional support for this view comes from Bruner, Mandler, O'Dowd, and Wallach (1958), who reported that learning of the "principle" type was impaired under high drive conditions.

Some investigators (Johnson, 1969; Lindholm \& Keesey, 1968; Stein \& Hearst, 1958) have proposed that ICS reinforcement may disrupt critical association processes which mediate learning. While at first this view might appear to explain the retarded reversal acquisition scores of those Ss receiving additional training (i.e., additional "disruptive" ICS), the average current intensity per $\mathrm{S}$ (.5 sec at 250 microamps) in the present experiment does not appear to be great enough to act in an analogous fashion to electroconvulsive shock. As in an investigation by Johnson (1968), no convulsions were observed in the present study at the relatively low current levels employed, and speculation about possible interference with consolidation processes at the neurophysiological level appears unjustified. The motivational impact of brain stimulation on cognitive capacity, however, remains to be explored.

\section{REFERENCES}

Bruner, J. S., Mandler, J. M., O'Dowd, D., \& Wallach, M. A. The role of overlearning and drive level in reversal learning. Journal of Comparative \& Physiological Psychology, 1958, 51, 607-613.

Denenberg, V. H. Critical periods, stimulus input, and emotional reactivity: A theory of infantile stimulation. Psychological Review, 1964, 71, 335-351.

Gallistel, C. Motivating effects in self-stimulation. Journal of Comparative \& Physiological Psychology, 1966, 62, 95-101. 
Johnson. R. N. Effects of intracranial reinforcement intensity and distribution variables on brightness reversal learning in rats. Journal of Comparative \& Phỵsiological Psychology, 1968. 66. 422-426.

Johnson. R. N. Discrimination reversal learning in rats with food and intracranial stimulation. Paper presented at the meeting of the Eastern Psychological Association. Philadelphia, April 1969.

Keesey. R. E. Hypothalamic stimulation as a reinforcer of discrimination learning. Journal of Comparative \& Physiological Psychology: 1966. 62. 231-236.

Kling. J. W.. \& Matsumiva. Y. Relative reinforcement values of food and intracranial stimulation. Science. 1962. 135. 668-670.

Lindholm. E. P.. \& Keesey. R. E. Discrimination learning as a function of the duration of rewarding hypothalamic stimulation. Psychonomic Science. 1968, 10. 153-154.

Lindholm, E. P.. \& Keesey. R. E. Faster rates of discrimination learning with centrally elicited reinforcement than with food. Journal of Comparative \& Phýsiological Psychology: 1970, 72. 318-327.

Mackintosh. N. J. Selective attention in animal discrimination learning. Psychological Bulletin, 1965, 64. 124-150.

Mackintosh, N. J. Further analysis of the overtraining reversal effect. Journal of Comparative \& Phýsiological Psychology, 1969. 67(2. Whole No. 2).

Reid. L. S. The development of noncontinuity behavior through continuity learning. Journal of Experimental Psychology, 1953. 46. 107-112.

Stein, L.. \& Hearst, E. Inhibitory effect of positively reinforcing brain stimulation on learning. American Psychologist. 1958. 13. 408. (Abstract)

(Received for publication June 8, 1973: revision received July $23,1973$. ) 